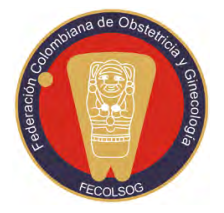

Revista Colombiana de Obstetricia y Ginecología Vol. 71 No. 3 • Julio-Septiembre 2020 • (275-285)

\title{
ANOMALÍAS GENITALES: CONTEXTUALIZACIÓN DE UIN CAMPO OLVIDADO EN EL DIAGNÓSTICO PRENATAL
}

\section{Grenital abnormallities: Contextuallization of a neglected area in prenatal diagnosis}

\author{
Álvaro López-Soto MD, PhD. ${ }^{1}$ \\ Recibido: 13 de noviembre de 2019/Aceptado: 18 de agosto de 2020
}

\section{RESUMEN}

Objetivo: hacer una reflexión sobre el bajo desarrollo que hay actualmente en el campo del diagnóstico prenatal de las anomalías genitales.

Materiales y métodos: a partir de la tesis de que el desarrollo del diagnóstico antenatal de las anomalías genitales es escaso, se presenta una comparación con el estado actual de otros campos del diagnóstico prenatal, así como con su contrapartida posnatal; se analizan las distintas causas que pueden haber llevado a esta situación, y se reflexiona sobre formas de mejora de la especialidad.

Conclusión: en comparación con otras áreas del diagnóstico prenatal, la detección de anomalías genitales tiene un menor nivel de desarrollo en cuanto a la disponibilidad de herramientas diagnósticas, de protocolos de manejo o investigación clínica. Algunas causas probables son una impresión de baja prevalencia, una importancia limitada o las dificultades para su exploración. Una forma de reforzar este componente de la medicina fetal sería la integración del conocimiento actual, la adquisición

* Correspondencia: Álvaro López Soto, C/ Puerta Nueva $374^{\circ} \mathrm{B} 30008$, Murcia (España). Teléfono: +34 679241077. alvarolopezsoto1@gmail.com

Unidad de Diagnóstico Prenatal, Hospital General Universitario Santa Lucía, Cartagena (España). de herramientas adecuadas, y una traslación a la medicina clínica.

Palabras clave: desarrollo sexual; diagnóstico prenatal; ultrasonografía; enfermedades fetales.

\section{ABSTRACT}

Objective: To reflect on how the area of genital abnormalities has fallen behind in prenatal diagnosis. Materials and methods: Based on the thesis that prenatal diagnosis of genital abnormalities has scarcely developed, a comparison with other areas of prenatal diagnosis and with its postnatal counterpart is presented; different explanations for this situation are examined; and a reflection is made on ways to improve the specialty.

Conclusion: Compared to other disciplines, prenatal diagnosis of genital abnormalities finds itself lagging behind in terms of diagnostic tools, management protocols and scientific literature. Potential causes include a perception of low prevalence and limited importance, or exploration challenges. Integration of current knowledge, together with the acquisition of the appropriate tools and translation to clinical medicine, would be a way to make this discipline stronger.

Key words: Sexual development; prenatal diagnosis; ultrasonography; fetal diseases. 


\section{INTRODUCCIÓN}

Bajo el nombre de anomalías genitales se definen una serie de condiciones que se caracterizan por alteraciones morfológicas en los órganos reproductivos tanto masculinos como femeninos (1). El diagnóstico de estas entidades se ha iniciado clásicamente en el recién nacido y continúa durante la infancia y la adolescencia (2). El manejo y tratamiento dependerán del tipo de entidad, este suele ser complejo y requerir la participación de múltiples especialidades como pediatría, endocrinología o urología, entre otros (3).

El uso del ultrasonido ha significado un importante avance en la medicina moderna ya que es una técnica no invasiva que permite el diagnóstico de alteraciones morfológicas en el sujeto. Su aplicación en el campo de la obstetricia representó un importante avance en medicina fetal, dado que permitió el diagnóstico de anomalías en el crecimiento, bienestar y presentación fetal, o de anomalías congénitas del sistema nervioso, cardiovascular, urinario o vascular, algunas de las cuales pueden ser incluso tratadas in útero, con una importante mejora en el pronóstico neonatal como, por ejemplo, las bridas congénitas, el mielomeningocele o la hernia diafragmática (4).

Pese a los grandes avances del ultrasonido en otros campos del diagnóstico prenatal, llama la atención el escaso desarrollo e impacto que ha tenido en el diagnóstico precoz de anomalías genitales. Como ejemplo tendríamos que en el año 2005, durante una conferencia de trabajo en Chicago (5), se definieron los principios y la formación del equipo multidisciplinar para el manejo de pacientes con anomalías del desarrollo sexual (ASD), que incluía la genética, la psicología clínica, así como la ginecología en su papel de cirugía correctiva. Sin embargo, en el documento no se hace referencia al papel del obstetra, la ecografía obstétrica o el diagnóstico prenatal de anomalías genitales. Esta primera gran ausencia se ha ido replicando en los años posteriores en multitud de guías y consensos en las que hay escasas menciones al papel del ultrasonido obstétrico en el diagnóstico prenatal de anomalías genitales (3,6-8). Todas estas situaciones podrían ser resultado del poco desarrollo del ultrasonido obstétrico en el diagnóstico de las ADS.

El diagnóstico prenatal de las anomalías genitales mediante el uso de ultrasonido (6) podría incluir la detección in utero de alteraciones morfológicas tales como la clitoromegalia, el micropene, el escroto bífido o criptorquidia (7). Su presencia, además, podría orientar sobre otras alteraciones más complejas, como el compromiso del aparato urinario, el sistema hormonal, el desarrollo sexual o la existencia de síndromes genéticos, así como también el uso de pruebas complementarias como las genéticas u hormonales; igualmente, podría llevar al establecimiento de manejos prenatales protocolizados y colaboración con diversas especialidades $(6,8,9)$. En definitiva, se trataría de un campo de estudio más amplio y complejo de lo que principio pudiera parecer.

En este artículo de reflexión nuestro objetivo es sustentar la tesis de que el diagnóstico prenatal de anomalías genitales tiene un escaso desarrollo, tanto en el uso de ultrasonidos como otras pruebas complementarias, y plantear hipótesis sobre las formas de mejorarla y sus ventajas. Para ello, comenzaremos con un análisis de la situación actual mediante la comparación con otros campos del diagnóstico prenatal y su contrapartida posnatal; verificaremos las posibles causas que han podido llevar a esta situación; señalaremos los beneficios que se podrían obtener con un adecuado desarrollo, y valoraremos las posibles maneras de potenciarlo.

\section{SITUACIÓN ACTUAL}

Para medir el nivel de desarrollo del diagnóstico prenatal de anomalías genitales nos proponemos hacer una la comparación con otros campos del diagnóstico prenatal, así como con su contrapartida, el diagnóstico posnatal de las ADS.

Comparación con los otros campos del diagnóstico prenatal. Para hacer este parangón utilizaremos las 
siguientes variables: existencia de medidas y tablas de referencia; descripción y registro de fenotipos y variantes patológicas; disponibilidad de protocolos y guías clínicas que incluyen recomendaciones para el diagnóstico prenatal por ultrasonido, y número y tipo de estudios que evalúan la exactitud o concordancia del ultrasonido en esa aplicación.

Medidas y tablas de referencia: en distintos campos del diagnóstico prenatal se dispone de mediciones estandarizadas y tablas de referencia actualizadas, por ejemplo, en patología congénita cardiaca (9), esquelética (10) o renal (11). Respecto de las anomalías genitales existen algunos trabajos sobre morfometría del aparato genital como la longitud del pene y la circunferencia escrotal $(2,12)$, el diámetro bilabial (13) o el tamaño testicular (14). Sin embargo, cada estudio utiliza mediciones diferentes y, hasta la fecha, aunque han intentado introducir estándares (15-17), no se ha tenido éxito en homologarlos.

Descripción y registro de fenotipos y variantes patológicas: se han estudiado y descrito extensamente las variantes de la "normalidad y anormalidad", y las características del desarrollo embrionario y fetal de otros sistemas, así como también su evolución a lo largo del tiempo en anomalías congénitas. Por ejemplo, tenemos las clasificaciones establecidas para los tipos de malformaciones broncopulmonares (18) o la precisión minuciosa en las evaluaciones ecográficas del desarrollo cardiaco (19) o cerebral del feto (20). En contraste, las anomalías genitales presentan un amplio rango de variabilidad clínica, tanto en anomalías leves como la clitoromegalia, como en defectos complejos y sindrómicos como la cloaca persistente (21). No existen registros, descripciones o clasificaciones detalladas, una de las principales cuestiones por resolver es la distinción ecográfica de anomalías genitales entre los fenotipos leves y los más severos (22).

En cuanto a la existencia de guías clínicas o protocolos de manejo que incluyan el ultrasonido diagnóstico prenatal, se dispone de manejos estan- darizados para, por ejemplo, la transfusión feto-fetal (23), la hernia diafragmática congénita (24) o la infección por parvovirus B19 (25). En comparación, el documento más importante del que se dispone respecto a las anomalías genitales sería un editorial sobre la aproximación diagnóstica publicado hace una década (1). Aparte del mismo, existen muy pocos trabajos que hayan sugerido algoritmos de diagnóstico o manejo prenatal $(26,27)$.

Por último, existen abundantes estudios de patologías fetales mucho menos prevalentes, como las anomalías de extremidades (28) o la gastrosquisis (29), o la realización de trabajos con diseños más complejos como los del grupo de colaboración del registro "lower urinary tract obstruction (LUTO)” (30) o el esfuerzo histórico que supuso el estudio "Management of Myelomeningocele Study (MOMS)” (31). Si evaluamos el número y tipo de estudios sobre anomalías genitales, la gran mayoría de bibliografía está formada por reportes de casos clínicos (32-34) o bien estudios de tipo retrospectivo que utilizan cohortes pediátricas (22). Otro ejemplo sería revisar algunos de los principales libros de texto de medicina fetal para observar que el capítulo correspondiente a patología genital apenas cubre unas pocas páginas $(35,36)$.

Comparación con el diagnóstico posnatal de anomalías genitales: el diagnóstico posnatal dispone de una amplia bibliografía que abarca libros de texto (37), guías clínicas $(3,38)$, documentos de consenso $(6,39)$, técnicas quirúrgicas $(40,41)$ e incluso redes de investigación internacionales (42).

\section{CAUSAS DEL ESCASO}

\section{DESARROLLO DEL ESTUDIO DE LAS ANOMALIIAS GENITALES}

En este apartado exploraremos las posibles causas del escaso desarrollo de este campo del diagnóstico prenatal.

Percepción de una baja prevalencia: si una patología tuviera una baja o muy baja prevalencia en la población, sería probable que recibiera igualmente una 
escasa atención. Fuchs et al. (22) comentan que la identificación prenatal de anomalías genitales continúa siendo un hallazgo raro en comparación con otros tipos de defectos. Sin embargo, es un evento frecuentemente diagnosticado en la etapa posnatal. Se ha informado que, en Escocia, la prevalencia de genitales ambiguos era de 5,8 x 1.000 recién nacidos en 1996 (43), y de 1 de cada 5.000 partos en Alemania para para los años 2000 a 2002 (44). En 2017, el equipo de Zarante publicó un análisis de los patrones de prevalencia de hipospadias en Sudamérica, y mostró una prevalencia de 1,13 de cada 1.000 partos y un incremento total del 3,3\% a lo largo de las últimas dos décadas (45). Paulozzi informa que la primera y segunda anomalía congénita más frecuente en Estados Unidos en el recién nacido varón sería la criptorquidia y la hipospadias, respectivamente (46). Blackless et al., por su parte, estima que la frecuencia de cualquier tipo de anomalía genital en recién nacidos estaría cercana al 2\% (47). Por tanto, no parece que sea tanto un problema de baja prevalencia, sino de falla en el diagnóstico previo al nacimiento.

Idea de una importancia limitada: la criptorquidia y los hipospadias, a pesar de ser frecuentes, suelen ser patologías aisladas, con un manejo posnatal muy estandarizado y buen pronóstico $(48,49)$. Sin embargo, muchas de las ADS se acompañan de otras malformaciones congénitas y las ADS serían un marcador de otros problemas de tipo genético, hormonal, genitourinario, etc. Se ha afirmado que una anomalía genital va a estar asociada, en un 25$50 \%$ de los casos, al menos a otra anomalía, especialmente en patología del tracto urinario $(22,26)$; por ejemplo, el hispospadias se relaciona con 369 síndromes reconocidos según en la base de datos de dismorfología de Winter y Baraitser (50). Por tanto, la importancia de las anomalías genitales no se basa exclusivamente en las alteraciones anatómicas que producen, sino en su papel como posibles marcadores de síndromes congénitos complejos.
Dificultad en la exploración: el examen clínico se considera el pilar básico del manejo de la patología genital y se han desarrollado diferentes técnicas, escalas y sistemas de puntuación para realizarlo (51). Una razón clara por la que los neonatólogos realizan la mayoría de los diagnósticos de anomalías genitales es el fácil acceso durante la exploración del recién nacido, lo que les permite reconocer la anatomía con todo detalle. Los neonatólogos, por ejemplo, realizan de manera estandarizada la medición de la longitud después de estirarlo (52), técnica que no puede ser aplicada en la etapa prenatal (15). Sin embargo, esta no es una cuestión que no pueda ser superada. La limitación en la exploración es inherente a todos los campos del diagnóstico prenatal, y la solución común ha sido el desarrollo de la tecnología ecográfica y una metodología estandarizada que nos permite examinar la anatomía en una aproximación similar al examen clínico (53). $\mathrm{Si}$ podemos estudiar detalles tan sutiles como las cisuras cerebrales (20) o la inserción de las válvulas cardiacas (19), probablemente podremos estudiar el aparato genital en mayor detalle. Un reciente estudio plantea la metodología de un nuevo tipo de corte ecográfico que permita la evaluación del sistema genitourinario y sus anomalías genitourinarias (54).

\section{IMPORTANCIA DE LA DETERMINACIÓN DE SEXO FETAL}

La determinación del sexo fetal ha sido uno de los aspectos más controversiales desde los inicios del diagnóstico prenatal por ultrasonido (55). La enorme importancia y expectación que se le da al sexo del feto incluye aspectos sociales, culturales, éticos y legales. Por ejemplo, en países asiáticos como China o India está prohibido informar sobre el sexo en pruebas genéticas prenatales por el fenómeno de abortos selectivos (56), en tanto Occidente debate sobre la diferencia entre sexo y género y el determinismo en los roles de género que puede generar 
esta información (57). En el caso de Occidente, el interés especial por conocer el sexo fetal antes del parto y lo más temprano posible llevó a un desarrollo significativo de los medios para realizarlo; actualmente, mediante ultrasonografía se puede conseguir una sensibilidad en segundo trimestre del 98-100\% (58), e incluso tasas similares en la ecografía de primer trimestre (59). Es más, la introducción en los últimos años del ADN fetal libre en sangre materna está permitiendo determinar el sexo de manera fiable a las pocas semanas del embarazo (60).

Podemos suponer que todo este interés sobre establecer el sexo fetal (tanto para conocerlo como para evitarlo) está monopolizando la atención sobre la evaluación de la existencia de posibles anormalidades. Se puede llegar a asumir que la visualización de un pene (determinante de sexo masculino) es un proceso equivalente a su estudio anatómico y al descarte de anomalías morfológicas, cuando no es así. En este sentido, dos documentos nos muestran cómo la importancia que se le da al sexo fetal ha desplazado la evaluación anatómica. Por un lado, la guía de examen de ecografía de segundo trimestre de la International Society of Ultrasound for Obstetrics and Gynaecology (ISUOG) indica, en el apartado dedicado a los genitales, que la determinación del sexo fetal no es obligatoria y que debe tenerse el consentimiento de los padres (53). Sorprende que sea la única parte en todo el documento en la que se discuten estos aspectos legales. En el caso de la "Executive Summary on Fetal Imaging", publicada en 2014 y firmada por las principales sociedades científicas estadounidenses, se estandariza la ecografía de manera que la determinación del sexo fetal se realice "solo en gestaciones múltiples y cuando esté medicamente indicada”. Los genitales ni siquiera se mencionan (61). Por tanto, la importancia del sexo fetal podría estar monopolizando la atención recibida a los genitales fetales tanto en los exámenes rutinarios como en los estudios de investigación. A diferencia de órganos como el corazón fetal donde el objetivo es conseguir la mejor forma de examen y evaluación, el campo del diagnóstico prenatal de anomalías genitales parecería estar más centrado en implicaciones ético-legales sobre si observar o no la presencia de un pene frente a su observación en busca de anomalías y malformaciones.

\section{BENEFICIOS DEL DIAGNÓSTICO PRENATAL DE ANOMALÍAS GENITALES}

De manera general, la utilidad del cribado sistemático de anomalías prenatales se encuentra establecida y consensuada desde hace más de veinte años (62). Más allá del cribado la detección temprana de patologías fetales permite diversas opciones como el tratamiento intraútero, un manejo que mejore los resultados perinatales, el consejo genético, la derivación precoz a centros especializados o la posibilidad de interrupción de la gestación (63).

Ya en el campo de las ADS, su diagnóstico prenatal va a recoger todos esos preceptos. Patologías como la hiperplasia suprarrenal congénita, que causa virilización del feto femenino y que puede ser tratada con corticosteroides (64), y en los casos de formas severas de esta condición, se pueden remitir a centros de referencia debido a la posibilidad de crisis adrenérgica al nacimiento, una emergencia endocrina que pone en riesgo la vida del recién nacido (65). Las malformaciones del tracto genitourinario como hipospadias o persistencia de valvas uretrales requieren un seguimiento periódico ante la posibilidad de desarrollar una obstrucción tardía que pueda afectar gravemente al desarrollo de los riñones y pulmones (66); hallazgos como un micropene pueden ser marcadores de patologías más complejas e importantes como aneuploidías fetales (T21, XXY, XXX), mutaciones genéticas (síndrome de Noonan, síndrome de Prader-Willi, síndrome de Robinow) y patologías del desarrollo hormonal (déficit de hormona del crecimiento, síndrome de hidantoína fetal, deficiencia de 5 - $\alpha$-reductasa) (67); en los casos que se considere pertinente se tiene la posibilidad de interrumpir la 
gestación en aquellas patologías con mal pronóstico o con una importante morbilidad (megalouretra congénita, extrofia cloacal, síndromes genéticos), posibilidad que está recogida en las guías clínicas de las principales sociedades ginecológicas $(68,69)$.

Existe otro beneficio adicional relacionado con el diagnóstico prenatal de genitales ambiguos y la asignación de sexo. En patologías como disgenesia gonadal mixta o síndrome de insensibilidad a andrógenos, puede darse el nacimiento de un bebé en el que no sea posible establecer su sexo de manera clara. Se trata de una situación traumática tanto para los padres como para el propio equipo médico, incluso con implicaciones legales en algunos países por no poder completar el certificado de nacimiento (70,71); pese a la existencia de protocolos urgentes de estudio o recomendaciones tales como dirigirse al neonato en términos neutros o elegir un nombre válido para ambos sexos (72), sigue siendo una situación difícil de manejar para los padres por el desconocimiento y las propias expectativas 73). Un diagnóstico prenatal de esta condición, con base en la determinación del cariotipo masculino y genitales femeninos (74) permite realizar un estudio de manera más programada y proporcionar un mejor consejo a los padres que les permita entender la situación.

\section{CÓMO MEJORAR EL DIAGNÓSTICO PRENATAL DE LA ADS}

Hemos visto que sigue habiendo esfuerzos por impulsar su desarrollo y que existe un potencial de mejora que debemos aprovechar, como pueden ser los diferentes intentos de introducción de medidas para biometría genital, la organización de un registro de variables patológicas o las propuestas de algoritmos de trabajo $(15,22,26)$. ¿Cómo podríamos mejorar esta situación? El objetivo sería dotar al campo prenatal de las anomalías genitales de un cuerpo de conocimientos sólido sobre el que desarrollarse. Nuestro planteamiento enfocaría los esfuerzos de mejora en tres fases: la integración del conocimiento actual, la adquisición de nuevo conocimiento y la traslación a la medicina clínica.

Como hemos visto, existen muchos trabajos desperdigados en la literatura científica a lo largo del tiempo en forma de editoriales, casos clínicos, cartas de investigación, e incluso estudios originales. Es posible encontrar casos prenatales de tumores testiculares malignos (75), dilataciones peneanas congénitas (76), discordancias entre el sexo cromosómico y ecográfico (77), torsiones testiculares intrauterinas (78), hipertrofias de labios y clítoris (79). Igualmente, disponemos de algunos documentos ya mencionados que sistematizan la información, como los editoriales y algoritmos de trabajo $(1,26,27)$. Todo este conocimiento debería de ser adecuadamente analizado e integrado mediante la realización de revisiones sistemáticas y sinopsis, e incluso esbozar protocolos y guías clínicas a partir de la información obtenida.

Hay varias herramientas de carácter básico de las que no se dispone en nuestra disciplina y que sería necesario establecer, como comentamos previamente. Incluiríamos entre nuestras necesidades las tablas de referencia, mediciones estandarizadas, cortes ecográficos o metodologías de trabajo. Ello requeriría, entre otros aspectos, la realización de estudios prospectivos con mediciones ecográficas seriadas de la anatomía genital femenina y masculina similares a algunos publicados $(2,79,80)$. Y dado que las guías ecográficas no hacen indicaciones sobre la metodología de trabajo (53), abordar propuestas como el corte perineal sagital medio (20) o desarrollar nuevas.

En última instancia, es necesario trasladar los conocimientos adquiridos a la práctica clínica y que estos no queden solo en estudios teóricos. Por un lado, hay que proporcionar al ultrasonografista ayuda diagnóstica mediante medidas estandarizadas o algoritmos de trabajo a la hora de evaluar casos seleccionados. Debe, incluso, plantearse la introducción rutinaria de ciertas medidas como la longitud del pene o el diámetro escrotal en las ecografías de segundo o trimestre, y, sobre todo, 
introducir un cambio de actitud sobre la evaluación de los genitales como órganos de estudio y no simples determinantes de sexo fetal. Por otra parte, es necesario compartir dichos conocimientos con las especialidades que tratan el diagnóstico posnatal, como pueden ser neonatología o urología, para complementar el manejo de las anomalías genitales y trabajar las líneas de investigación futuras. Por ejemplo, si la criptorquidia es la anomalía congénita más frecuente, y cuanto mayor tarde el diagnóstico mayores secuelas habrá en el testículo afectado $(80,81)$, ¿puede el diagnóstico prenatal ayudar a un menor deterioro o aportar información útil con vistas a la cirugía?

\section{CONCLUSIÓN}

En este artículo se presentan argumentos que validan la tesis de que el campo del diagnóstico prenatal de anomalías genitales tiene poco desarrollo en la actualidad, pese a ser un área relevante. Se requiere la integración del conocimiento actual, la estandarización de mediciones y técnicas para la medición de los genitales en el periodo antenatal y evaluar estas metodologías con los hallazgos neonatales para determinar su exactitud y real utilidad más allá de la tamización de las ADS.

\section{REFERENCIAS}

1. Chitayat D, Glanc P. Diagnostic approach in prenatally detected genital abnormalities. Ultrasound Obstet Gynecol. 2010;35(6):637-46. https://doi.org/10.1002/ uog.7679

2. Akpinar F, Yilmaz S, Akdag Cirik D, Kayikcioglu F, Dilbaz B, Yucel H, et al. Sonographic assessment of the fetal penile development. Fetal Pediatr Pathol. 2016;35(2):88-92. https://doi. org/10.3109/15513815. 2015.1135494

3. Ahmed SF, Achermann JC, Arlt W, Balen A, Conway G, Edwards Z, et al. Society for Endocrinology UK guidance on the initial evaluation of an infant or an adolescent with a suspected disorder of sex development (Revised 2015). Clin Endocrinol (Oxf). 2016;84(5):771-88. https://doi.org/10.1111/cen. 12857
4. Abinader R, Warsof SL. Benefits and pitfalls of ultrasound in obstetrics and gynecology. Obstet Gynecol Clin NA. 2019;46(2):367-78. https://doi. org/10.1016/j.ogc.2019.01.011

5. Hughes IA, Houk CP, Ahmed SF, Lee P. Consensus statement on management of intersex disorders. Pediatrics. 2006;118(2):e488-500. https://doi.org/ 10. 1542/peds.2006-0738

6. Lee PA, Nordenström A, Houk CP, Ahmed SF, Auchus R, Baratz A, et al. Global disorders of sex development update since 2006: Perceptions, approach and care. Horm Res Paediatr. 2016;085:158-80. https://doi. org/10.1159/000442975

7. Rolston AM, Gardner M, van Leeuwen K, Mohnach L, Keegan C, Vilain E, et al. Disorders of Sex Development (DSD): Clinical service delivery in the United States. AM J Med Genet C Semin Med Genet. 2017;175(2):268-78. https://doi.org/10.1002/ ajmg.c. 31558

8. Cools M, Nordenström A, Robeva R, Hall J, Westerveld P, Flück C, et al. Caring for individuals with a difference of sex development (DSD): A Consensus Statement. Nat Rev Endocrinol. 2018;14:415-29. https://doi.org/10.1038/s41574-018-0010-8

9. Krishnan A, Pike JI, Mccarter R, Fulgium AL, Wilson E, Donofrio MT, et al. Predictive Models for Normal Fetal Cardiac Structures. J Am Soc Echocardiogr. 2020;29(12):1197-206. https://doi.org/10.1016/j. echo.2016.08.019

10. Mark Curran. Skeletal Survey Calculator. Disponible en: http://perinatology.com/calculators/Skeletal Survey.html

11. Brennan S, Watson D, Rudd D, Schneider M, Kandasamy Y. Evaluation of fetal kidney growth using ultrasound: A systematic review. Eur J Radiol. 2017;96:55-64. https://doi.org/10.1016/j.ejrad.2017.09.017

12. Achiron R, Pinhas-Hamiel O, Zalel Y, Rotstein Z, Lipitz S. Development of fetal male gender: Prenatal sonographic measurement of the scrotum and evaluation of testicular descent. Ultrasound Obstet Gynecol. 1998;11(4):242-5. https://doi.org/10.1046/j.14690705.1998.11040242.x 
13. Nemec U, Weber M, Kasprian G, Krestan CR, Graham JM, Prayer D. Female external genitalia on fetal magnetic resonance imaging. 2011;38(6):695-700. https://doi.org/10.1002/uog.8973

14. Rotondi M, Valenzano F, Bilancioni E, Spano G, Rotondi M, Giorlandino C. Prenatal measurement of testicular diameter by ultrasonography: Development of fetal male gender and evaluation of testicular descent. Prenat Diagn. 2001;21(2):112-5. https:/doi. org/10.1002/1097-0223(200102)21:2<112:AID$\mathrm{PD} 2>3.0 . \mathrm{CO} ; 2-1$

15. Perlitz Y, Keselman L, Haddad S, Mukary M, Izhaki I, Ben-Ami M. Prenatal sonographic evaluation of the penile length. Prenat Diagn. 2011;31(October):1283-5. https://doi.org/10.1002/pd.2885

16. Pinette MG, Wax JR, Blackstone J, Cartin A. Normal growth and development of fetal external genitalia demonstrated by sonography. J Clin Ultrasound. 2003;31(9):465-72. https://doi.org/10.1002/ jcu. 10207

17. Nemec SF, Nemec U, Weber M, Kasprian G, Brugger PC, Krestan CR, et al. Male sexual development in utero: Testicular descent on prenatal magnetic resonance imaging. Ultrasound Obstet Gynecol. 2011;38(6):688-94. https://doi.org/10.1002/ uog. 8964

18. Kane SC, Ancona E, Reidy L. The utility of the congenital pulmonary airway malformation-volume ratio in the assessment of fetal echogenic lung lesions: A systematic review. Fetal Diagn Ther. 2020;47(3):171181. https://doi.org/10.1159/000502841

19. Ge S, Maulik D. Introduction: From fetal echocardiography to fetal cardiology : A journey of over half a century. Echocardiography 2017; 34(12):1757-1759. https://doi.org/10.1111/echo.13776

20. van der Knoop B, Zonnenberg I, Verbeke J, de Vries LS, Pistorius LR, van Weissenbruch MM, et al. Additional value of advanced neurosonography and magnetic resonance imaging in fetuses at risk for brain damage. Ultrasound Obstet Gynecol. 2020;56(3):348-58. https://doi.org/10.1002/uog.21943
21. Gilboa Y, Perlman S, Kivilevitch Z, Messing B, Achiron R. Prenatal anogenital distance is shorter in fetuses with hypospadias. J Ultrasound Med. 2017;36(1):17582. https://doi.org/10.7863/ultra.16.01006

22. Fuchs F, Borrego P, Amouroux C, Antoine B, Ollivier M, Faure JM, et al. Prenatal imaging of genital defects: Clinical spectrum and predictive factors for severe forms. BJU Int. 2019; 124(5):876-82. https://doi. org/10.1111/bju. 14714

23. Committee CS. ISUOG Practice Guidelines: Role of ultrasound in twin pregnancy. Ultrasound Obstet Gynecol. 2016;247-63. https://doi.org/10.1002/ uog. 15821

24. Kosinski P, Wielgos M. Congenital diaphragmatic hernia: Pathogenesis, prenatal diagnosis and management — literature review. Ginekol Pol. 2017;88(1):24-30. https://doi.org/10.5603/GP.a2017.0005

25. Ornoy A, Ergaz Z. Parvovirus B19 infection during pregnancy and risks to the fetus. Birth Defects Res. 2017; 15;109(5):311-23. https://doi.org/10.1002/ bdra. 23588

26. Pajkrt E, Petersen OB, Chitty LS. Fetal genital anomalies: An aid to diagnosis. Prenat Diagn. 2008; 28(5):389-98. https://doi.org/10.1002/pd.1979

27. Adam MP, Fechner PY, Ramsdell LA, Badaru A, Grady RE, Pagon RA, et al. Ambiguous genitalia: What prenatal genetic testing is practical? Am J Med Genet Part A. 2012;158 A(6):1337-43. https://doi.org/10.1002/ ajmg.a. 35338

28. Shi Y, Zhang B, Kong F, Li X. Prenatal limb defects: Epidemiologic characteristics and an epidemiologic analysis of risk factors. Medicine. 2018 Jul;97(29):e11471. https://doi.org/10.1097/MD.0000000000011471

29. Alam A, Sahu S, Indrajit IK, Sahani H, Bhatia M, Kumar R. Gastroschisis-antenatal diagnosis. Med J Armed Forces India. 2011;67(2):169-170. https:// doi.org/10.1016/S0377-1237(11)60026-9

30. Morris RK, Middleton LJ, Malin GL, Daniels J, Khan KS, Deeks J, et al. Outcome in fetal lower urinary tract obstruction: a prospective registry study. 2015:42431. https://doi.org/10.1002/uog.14808 
31. Adzick N, Thom E, Spong C, Brock J, Burrows PK, Johnson MP, et al. A randomized trial of prenatal versus postnatal repair of myelomeningocele. $\mathrm{N}$ Engl J Med. 2011;993-1004. https://doi.org/10.1056/ NEJMoa1014379

32. Sexton P, Thomas JT, Petersen S, Brown N, Arms JE, Bryan J, et al. Complete penoscrotal transposition: Case report and review of the literature. Fetal Diagn Ther. 2015;37(1):70-4. https://doi.org/10. 1159/000358592

33. Ochiai D, Omori S, Ikeda T, Yakubo K, Fukuiya T. A rare case of meconium periorchitis diagnosed in utero. Case Rep Obstet Gynecol. 2015;2015:1-2. https:/doi. org/10.1155/2015/606134

34. Inde Y, Terada Y, Ikegami E, Sekiguchi A, Nakai A, Takeshita T. Bifid scrotum and anocutaneous fistula associated with a perineal lipomatous tumor complicated by temporary bilateral cryptorchidism in utero mimicking ambiguous genitalia: 2-D/3-D fetal ultrasonography. J Obstet Gynaecol Res. 2014;40(3):843-8. https://doi.org/10.1111/jog.12232

35. Copel J, D’Alton M, Feltovich H, Gratacós E, Odibo A, Platt L, et al. Obstetric Imaging: Fetal Diagnosis and Care. Philadelphia: Elsevier Inc.; 2017.

36. Gratacós E, Figueras F, Martínez J. Fetal Medicine. 2nd ed. Pubns Z\& U, editor. Madrid: Editorial Panamericana; 2018.

37. Docimo S, Canning D, Khoury A, Pippi Salle JL. Textbook of Clinical Pediatric Urology. 6th ed. CRC Press; 2018.

38 Ministerio de Salud y Protección Social de Colombia - Colciencias. Guía de práctica clínica. Guía No. 03: Detección de anomalías congénitas en el recién nacido. Bogotá; 2013. Disponible en https://medicosgeneralescolombianos.com/images/Guias_2013/ gpc_03prof_sal_ac.pdf

39. Grimbizis GF, Di A, Sardo S, Saravelos SH, Gordts $\mathrm{S}$, Exacoustos C, et al. The Thessaloniki ESHRE/ ESGE consensus on diagnosis of female genital anomalies. Hum Reprod. 2016;31(1):2-7. https:// doi.org/10.1093/humrep/dev264
40. Manjunath KN, Venkatesh MS. M-Plasty for Correction of incomplete penoscrotal transposition. World J Plast Surg. 2014;3(2):138-41.

41. Theodoridis TD, Obstetrics A, Grimbizis GF. Best practice \& research clinical obstetrics and gynaecology surgical management of congenital uterine anomalies (including indications and surgical techniques). Best Pract Res Clin Obstet Gynaecol. 2020;59(2019):6676. https://doi.org/10.1016/j.bpobgyn.2019.02.006

42. I-DSD. I-CAH Registry; 2020. Disponible en: https:// home.i-dsd.org/

43. Ahmed SF, Dobbie R, Finlayson AR, Gilbert J, Youngson G, Chalmers J, et al. Prevalence of hypospadias and other genital anomalies among singleton births, 19881997, in Scotland. Arch Dis Child Fetal Neonatal Ed. 2004;89(2):149F-151. https://doi.org/10.1136/ adc. 2002.024034

44. They U, Lanz K, Holterhus PM, Hiort O. Epidemiology and initial management of ambiguous genitalia at birth in Germany. Horm Res. 2006;66(4):195-203. https://doi.org/10.1159/000094782

45. Fernández N, Pérez J, Monterrey P, Poletta FA, Bägli DJ, Lorenzo J, et al. ECLAMC Study. Prevalence patterns of hypospadias in South America: multinational analysis over a 24-year period. Int Braz J Urol. 2017;43(2):325-34. https://doi.org/10.1590/ s1677-5538.ibju.2016.0002

46. Paulozzi LJ. International trends in rates of hypospadias and cryptorchidism. Environ Health Perspect. 1999;107(4):297-302. https://doi.org/10.1289/ ehp.99107297

47. Blackless M, Charuvastra A, Derryck A, FaustoSterling A, Lauzanne K, Lee E. How sexually dimorphic are we? Review and synthesis. Am J Hum Biol. 2000;12(2):151-66. https://doi.org/10.1002/ (SICI) 1520-6300(200003/04) 12:2<151::AIDAJHB1>3.0.CO;2-F

48. Braga LH, Lorenzo AJ, Romao RLP. Canadian Urological Association-Pediatric Urologists of Canada (CUA-PUC) guideline for the diagnosis, management, and followup of cryptorchidism. Can Urol Assoc 
J. 2017;11(7):E251-60. https://doi.org/10.5489/ cuaj. 4585

49. Keays MA, Dave S. Current hypospadias management: Diagnosis, surgical management, and long-term patient-centred outcomes. Can Urol Assoc J. 2017;11(1-2Suppl1):S48-S53. https://doi. org/10.5489/cuaj.4386

50. Winter RM, Baraitser M . The London Dysmorphology Data- base. Oxford: Oxford University Press; 2001.

51. Davies JH, Cheetham T. Recognition and assessment of atypical and ambiguous genitalia in the newborn. Arch Dis Child. 2017;102(10):968-74. https://doi. org/10.1136/archdischild-2016-311270

52. Cheng PS, Chanoine JP. Should the definition of micropenis vary according to ethnicity? Horm Res. 2001;55(6):278-81. https://doi. org/10.1159/000050013

53. Salomon LJ, Alfirevic Z, Berghella V, Bilardo C, Hernandez-Andrade E, Johnsen SL, et al. Practice guidelines for performance of the routine mid-trimester fetal ultrasound scan. Ultrasound Obstet Gynecol. 2011;37(1):116-26. https://doi.org/10.1002/ uog. 8831

54. Garel J, Blondiaux E, Valle V Della, Guilbaud L, Khachab F, Jouannic J, et al. The perineal midsagittal view in male fetuses - pivotal for assessing genitourinary disorders. Pediatr Radiol. 2020;50(4):575-82. https://doi.org/10.1007/s00247-019-04551-w

55. Stocker J, Evens L. Fetal sex determination by ultrasound. Obstet Gynecol. 1977;50(4).

56. Mohapatra S. Global Legal Responses to Prenatal Gender Identification and Sex Selection. Nev. L. J. 2013;13(3):690.

57. Kessler SJ, McKenna W. Gender: An Ethnomethodological Approach. Chicago: University of Chicago Press; 1978.

58. Kearin M, Pollard K, Garbett I. Accuracy of sonographic fetal gender determination: Predictions made by sonographers during routine obstetric ultrasound scans. Australas J Ultrasound Med. 2015;17(3):12530. https://doi.org/10.1002/j.2205-0140.2014. tb00028.x
59. Colmant C, Morin-Surroca M, Fuchs F, Fernandez $\mathrm{H}$, Senat MV. Non-invasive prenatal testing for fetal sex determination: Is ultrasound still relevant? Eur J Obstet Gynecol Reprod Biol. 2013;171(2):197-204. https://doi.org/10.1016/j.ejogrb.2013.09.005

60. Akolekar R, Farkas DH, Vanagtmael AL, Bombard AT, Nicolaides KH. Fetal sex determination using circulating cell-free fetal DNA (ccffDNA) at 11 to 13 weeks of gestation. Prenat Diagn. 2010;30(10):918-23. https:// doi.org/10.1002/pd.2582

61. Reddy UM, Abuhamad AZ, Levine D, Saade GR. Fetal imaging: Executive summary of a joint Eunice Kennedy Shriver National Institute of Child Health and Human Development, Society for MaternalFetal Medicine, American Institute of Ultrasound in Medicine, American College of Obstetricians and Gynecolog. Obstet Gynecol. 2014;123(5):1070-82. https://doi.org/10.1016/j.ajog.2014.02.028

62. Grandjean H, Larroque D, Levi S. The performance of routine ultrasonographic screening of pregnancies in the Eurofetus Study. Am J Obstet Gynecol. 1999;181(2):446-54. https://doi.org/10.1016/ S0002-9378(99)70577-6

63. Temming LA, Macones GA. Seminars in Perinatology what is prenatal screening and why to do it? Semin Perinatol. 2020;40(1):3-11. https://doi.org/10.1053/j. semperi.2015.11.002

64. Al-Jurayyan NAM. Ambiguous genitalia: Two decades of experience. Ann Saudi Med. 2011;31(3):284-8. https://doi.org/10.4103/0256-4947.81544

65. Vats P, Dabas A, Jain V, Seth A, Yadav S, Kabra M, et al. Newborn Screening and Diagnosis of Infants with Congenital Adrenal Hyperplasia. Indian Pediatr. 2020;57(1):49-55. https://doi.org/10.1007/s13312020-1703-3

66. Haeri S. Fetal Lower Urinary Tract Obstruction (LUTO): A practical review for providers. Matern Heal Neonatol Perinatol. 2015;1:26. https://doi. org/10.1186/s40748-015-0026-1

67. Hatipoglu N, Kurtoglu S. Micropenis: Etiology, Diagnosis and Treatment Approaches. J Clin Res Pediatr Endocrinol. 2013;5(4):217-23. https://doi. org/10.4274/Jcrpe.1135 
68. National Institute for Health and Care Excellence. Abortion care. NICE Guideline (NG140). 2019. Disponible en: https://www.nice.org.uk/guidance/ng140

69. Committee on Health Care for Underserved Women. Committee Opinion: Abortion Training and Education. Obstet Gynecol. 2017;(612):1-5.

70. Oliveira MDS, Paiva-e-silva RB De, Guerra-junior G. Parents' experiences of having a baby with ambiguous genitalia. 2015;28:833-8. https://doi.org/10.1515/ jpem-2014-0457

71. Lathrop B, Cheney T, Hayman A. Ethical DecisionMaking in the Dilemma of the Intersex Infant. Issues Compr Pediatr Nurs. 2014;37(1):25-8. https://doi.or g/10.3109/01460862.2013.855842

72. Mccann-Crosby B, Sutton VR. Disorders of sexual development. Clin Perinatol. 2020;42(2):395-412. https://doi.org/10.1016/j.clp.2015.02.006

73. López A, Vázquez R, Rubio M, Lorente A, García O, Martínez J. La importancia del sexo fetal en la ecografía morfológica: genitales ambiguos y disgenesia gonadal mixta. Prog Obstet Ginecol. 2017;60(5):474-9.

74. Franasiak JM, Yao X, Ashkinadze E, Rosen T, Scott RT. Discordant embryonic aneuploidy testing and prenatal ultrasonography prompting androgen insensitivity syndrome diagnosis. Obstet Gynecol. 2015;125(2):3836. https://doi.org/10.1097/AOG.0000000000000503

75. Peterson C, Skoog S. Prenatal diagnosis of juvenile granulosa cell tumor of the testis. J Pediatr Urol. 2008;4(6):472-4. https://doi.org/10.1016/j.jpurol.2008.04.005
76. Moaddab A, Sananes N, Hernandez-Ruano S, Britto ISW, Blumenfeld Y, Stoll F, et al. Prenatal diagnosis and perinatal outcomes of congenital megalourethra: A multicenter cohort study and systematic review of the literature. J Ultrasound Med. 2015;34(11):2057-64. https://doi.org/10.7863/ultra.14.12064

77. Scibetta EW, Gaw SL, Rao RR, Silverman NS, Han CS, Platt LD. Clinical accuracy of abnormal cell-free fetal DNA results for the sex chromosomes. Prenat Diagn. 2017;37(13):1291-7. https://doi.org/10.1002/ pd. 5146

78. van der Sluijs JW, den Hollander JC, Lequin MH, Nijman RM, Robben SGF. Prenatal testicular torsion: Diagnosis and natural course. An ultrasonographic study. Eur Radiol. 2004;14(2):250-5. https://doi. org/10.1007/s00330-003-2019-0

79. Zimmer E, Blazer S, Blumenfeld Z, Bronshtein M. Fetal transient clitoromegaly and transient hypertrophy of the labia minora in early and mid pregnancy. J Ultrasound Med. 2012;(31):409-15. https://doi. org/10.7863/jum.2012.31.3.409

80. Vuillard E, Chitrit Y, Dreux S, ElGhoneimi A, Oury J, Muller F. Sonographic measurement of corpus spongiosum in male fetuses. Prenat Diagn. 2011;31:11603. https://doi.org/10.1002/pd.2854

81. Tasian GE, Hittelman AB, Kim GE, DiSandro MJ, Baskin LS. Age at orchiopexy and testis palpability predict germ and leydig cell loss: Clinical predictors of adverse histological features of cryptorchidism. J Urol. 2009;182(2):704-9. https://doi.org/10.1016/j. juro.2009.04.032 